43.

\section{Prehospital Determination of Eligibility for Thrombolytic Therapy}

T Delozier, $M D^{*}$, LJ White, MS, JM Dougherty, MD, and the Summit County Prehospital Research Group

The Emergency Medicine Residency Program, Akron General Medical Center, Akron, Ohio USA

Purpose: To determine the degree of concordance between thrombolytic checklists completed by emergency medical technicians (EMTs) in the field and corresponding checklists completed by emergency physicians in the emergency department (ED).

Methods: This was a comparative analysis of matched pairs of thrombolytic checklists. Subjects included patients 35 years or older with clinical signs or symptoms of cardiac origin who were transported to the ED by ambulance. The checklist contained 12 items and was completed by EMTs during transport of eligible patients. An identical checklist was completed in the ED by an emergency physician upon arrival of the patient. The main outcome measurement was concordance between the matched pairs.

Results: Fifty-five matched pairs of checklists were compared. Mean age of the subjects was 66 years; range 35-91. Mean duration of chest pain prior to contacting EMS was 9.6 hours; range $10 \mathrm{~min}$. to 72 hours. The median concordance score between matched pairs of checklists was $93 \%$. Discordant items included current blood pressure readings, and assessment of possible aortic dissection. Maximum agreement was scored on items regarding recent surgery and trauma, and current pregnancy. Participating EMTs possessed a mean of eight years of EMS service. Emergency physicians completing the checklists included resident physicians $(73 \%)$ and attending physicians (27\%). Seven subjects $(14 \%)$ received thrombolytic therapy while in the ED.

Conclusion: There was excellent agreement between prehospital and ED checklists. Combined with prehospital telemetry, thrombolytic eligibility screening has the potential to speed up delivery of thrombolytic agents for patients who need them.
46.

The Accuracy of Medical Records and Police Reports in Determining Motor Vehicle Crash Characteristics

Robert J. Grant, MD, * Mary Ann Gregor, MHSA, Ronald F. Maio, $D O, M S$, Shaun S. Huang, MD

Section of Emergency Medicine, Department of Surgery, University of Michigan, Ann Arbor, Michigan USA

Objective: To determine the accuracy of ambulance (AR), emergency department (EDR) and police $(\mathrm{PR})$ records in describing motor vehicle crash (MVC) characteristics when compared to a standard - an in-depth motor vehicle crash investigation record (CIR).

Methods: Fifty-six MVC patients transported to a suburban, university hospital emergency department via ambulance and included in a crash investigation were identified. The time period was January 1993 through December 1995. Data sources were the AR, EDR, PR and CIR. The CIR was abstracted to a standard form. The other data sources were abstracted to a standard form using a retrospective, blinded review. Variables included occupant position, restraint use, air bag deployment, type of impact, ejection, and external cause of injury code. Accuracy was measured by determining percent agreement and Kappa for each data source compared to the CIR.

Results: Forty-six cases $(82 \%)$ had one or more episodes of discordance. The mean percent agreement for the sources studied was AR 0.813, EDR 0.893 and PR 0.932. Mean values for Kappa were AR 0.712, EDR 0.831 and PR 0.885. Among variables, restraint use was determined with the least accuracy with means for percent agreement and Kappa of 0.792 and 0.674 , respectively. For the $\mathrm{AR}$, discordance was due to missing information almost $50 \%$ of the time.

Conclusions: The inaccuracy among data sources in determining MVC characteristics varies. The AR is the least accurate data source for crash information. Injury control researchers need to consider how to improve data source accuracy. 